\title{
Cataract surgery in Fuchs' heterochromic iridocyclitis
}

\author{
Derek R Sherwood, A Ralph Rosenthal
}

\begin{abstract}
Eighteen eyes in 17 patients with Fuchs' heterochromic iridocyclitis underwent cataract extraction with or without intraocular lens implantation (17 extracapsular and one intracapsular). Intraoperative complications included hyphaema, poor pupillary dilatations, and localised zonule dehiscence with vitreous loss. Only four eyes developed a marked anterior uveitus (two pseudophakic and two aphakic) which resolved within 2 weeks with topical steroids. Three eyes developed a rise in intraocular pressure (IOP) to more than $30 \mathrm{~mm} \mathrm{Hg}$ on the first postoperative day. In all three eyes the IOP returned to normal off all therapy within 1 week. In one of three eyes preoperative glaucoma was made worse following surgery. Visual acuity testing revealed that 15 eyes $(83.3 \%)$ achieved $6 / 12$ vision or better. Lamellar macular hole, pre-existing macular scar, and pre-existing retinal detachment accounted for the poor visual result.
\end{abstract}

Fuchs' heterochromic iridocyclitis (FHIC) was first clearly defined by Ernest Fuchs in $1906 .^{1}$ This often overlooked uveitis syndrome is characterised by chronic low grade anterior chamber and anterior vitreous inflammation, diffusely distributed stellate keratic precipitates (KPs), full thickness iris atrophy, posterior subcapsular cataract, and abnormal iris blood vessels. ${ }^{2}$ FHIC is usually unilateral ${ }^{3}$ and progressive iris stromal atrophy leads to heterochromia in most patients. The visual outcome following cataract surgery in patients with FHIC has been the subject of several conflicting reports. ${ }^{4-9}$ Until recently little information about visual outcome, complications during surgery, and the role of posterior chamber intraocular lens (IOL) implantation following extracapsular cataract extraction (ECCE) has been available. ${ }^{111} \mathrm{We}$ present the findings of a study on patients with FHIC undergoing cataract extraction with or without IOL implantation.

\section{Patients and methods}

All patients with FHIC undergoing cataract surgery at Leicester Royal Infirmary between March 1988 and January 1990 were included in the study. Eighteen eyes of 17 patients were examined preoperatively and the diagnosis of FHIC made according to the presence of standard accepted major and minor criteria. ${ }^{312}{ }^{13} \mathrm{~A}$ record of preoperative use of glaucoma medication and systemic steroids was made and the intraocular pressure (IOP) measured.

Surgery was performed on the 18 eyes by six different surgeons. Twelve eyes were operated on by one of the authors (DRS), and in these patients a particular note was made of complications during surgery including: anterior chamber haemorrhage, poor pupillary dilatation and zonule dehiscence, or posterior capsule tears. Eleven women and six men with ages ranging from 22-77 years underwent surgery. One female patient had bilateral cataract extractions and two patients had previously had cataract extraction on the other eye. Three eyes were being treated for glaucoma with topical medications preoperatively. Seventeen eyes had ECCE and one eye with a subluxed lens had an intracapsular cataract extraction (ICCE) and anterior chamber IOL implanted. Of the 17 eyes having ECCE, five were implanted with posterior chamber IOLs. The decision to implant an IOL was made preoperatively by the surgeon after discussion with the patient. In all patients an aqueous sample was aspirated before anterior capsulotomy and a peripheral iridectomy performed. Betamethasone $(2 \mathrm{mg}$ ) and gentamicin base $(20 \mathrm{mg}$ ) were administered subconjunctivally at completion of the surgical procedure. Three patients received preoperative systemic steroids which was continued for 2 weeks following surgery.

After the surgery all patients were treated with hourly topical steroids, topical antibiotics four times daily, and cycloplegics twice daily. All patients were examined postoperatively. Visual acuity was measured with the patients wearing spectacle or contact lens correction. Patients were asked whether they were wearing their contact lens regularly if unilaterally aphakic. The necessity for YAG laser capsulotomy was noted as was any vitreous or retinal pathology. Follow-up ranged from 3 months to 24 months with an average follow-up of 13 months.

\section{PERIOPERATIVE COMPLICATIONS}

All 12 eyes operated on by DRS had ECCE. One of these eyes was implanted with a one-piece poly(methylmethacrylate) (PMMA) posterior chamber IOL. Haemorrhage arising from the peripheral iris was noted in all 12 eyes at the time of paracentesis. The blood partially obscured the capsule during capsulotomy in five eyes but was cleared from the central anterior chamber with the use of sodium hyaluronate. Poor pupillary dilatation was noted in four eyes all of which were noted preoperatively to have marked iris stromal atrophy. Localised zonule dehiscence with vitreous loss occurred in one eye.

\section{POSTOPERATIVE COMPLICATIONS}

Postoperative hyphaema was not seen in any eye. Four of the 18 eyes developed a marked anterior 
uveitis despite hourly topical steroids. Two of these eyes had been implanted with a posterior chamber IOL and two were aphakic. The uveitis resolved over 1-2 weeks without requiring periocular steroid injection or systemic steroids. None of the three patients receiving preoperative systemic steroids developed significant uveitis. Three eyes developed a rise in IOP to more than $30 \mathrm{~mm} \mathrm{Hg}$ on the first postoperative day. One of the eyes was already being treated for glaucoma. In all three eyes the IOP returned to normal within 1 week and only required short term treatment with oral acetazolamide. One eye with well controlled glaucoma preoperatively had a rise in IOP 3 weeks postoperatively and has required increased treatment since then (followup 14 months). All IOLs were noted to have some KPs on the surface but these were not considered to interfere with visual acuity. Seven eyes were noted to have stellate KPs on the posterior capsule. Three eyes subsequently required YAG laser capsulotomy, two of which were aphakic and one pseudophakic with a posterior chamber IOL.

\section{VISUAL OUTCOME}

The visual outcome is given in Table 1 .

Fifteen eyes $(83.3 \%)$ achieved $6 / 12$ vision or better. Three eyes had vision worse than $6 / 12$. Of these one eye was found during surgery to have a pre-existing subtotal rhegamatogenous retinal detachment which was later reattached by vitrectomy and silicone oil but visual acuity remained at hand movements at 0.5 metre. One eye with visual acuity of $6 / 18$ was found to have a lamellar macular hole and one eye with visual acuity of $6 / 60$ had dense vitreous opacities and had a known macular scar preoperatively. Vitreous opacities were noted in all eyes postoperatively but were least apparent in the three eyes with $6 / 6$ or better visual acuity.

\section{CONTACT LENS WEAR}

Eight patients were unilaterally aphakic and six of these patients had visual acuity of $6 / 12$ or better with a corrective contact lens. Only two of these patients were regularly wearing their contact lenses.

\section{Discussion}

Decisions regarding cataract surgery in patients with FHIC are often influenced by the relatively young age of the patients, the poor experience with cataract surgery in other forms of uveitis, and the tendency of the disease to be unilateral. However the condition may be bilateral as one patient in this series who had bilateral FHIC

Table 1 Best corrected operative visual acuity following cataract extraction in 18 eyes with Fuchs' heterochromic iridocyclitis

\begin{tabular}{lllll}
\hline $\begin{array}{l}\text { Snellen } \\
\text { acuity }\end{array}$ & $\begin{array}{l}\text { Pseudophakic } \\
\text { eyes }\end{array}$ & $\begin{array}{l}\text { Aphakic } \\
\text { eyes }\end{array}$ & Total & $\begin{array}{l}\text { Total } \\
\text { cumulative \% }\end{array}$ \\
\hline $6 / 5-6 / 6$ & 2 & 1 & 3 & $16 \cdot 6$ \\
$6 / 6-6 / 9$ & 1 & 6 & 7 & $55 \cdot 5$ \\
$6 / 9-6 / 12$ & 2 & 3 & 5 & $83 \cdot 3$ \\
$6 / 12-6 / 24$ & 1 & - & 1 & $89 \cdot 0$ \\
$6 / 24$ or worse & - & 2 & 2 & $100 \cdot 0$ \\
\hline
\end{tabular}

underwent cataract surgery. The occurrence of the condition binocularly has been reported before. In fact three of the 38 original cases described by Fuchs had slight inflammatory changes in the normal darker eye. ${ }^{1}$ More recently in a series of 54 Caucasian patients four $(7 \%)$ had bilateral involvement. ${ }^{12}$

Ward and Hart $^{6}$ suggested that there was a high incidence of hyphaema, vitreous haemorrhage, and glaucoma following ICCE in patients with FHIC. More recently Smith and O'Connor ${ }^{7}$ in a retrospective review of 29 cases found cataract extraction in FHIC to be only 'slightly more hazardous' than in routine cases. The use of IOLs in FHIC has been given support by Mooney and O'Connor ${ }^{4}$ and by Mills and Rosen ${ }^{5}$ who found few problems associated with iris fixated IOLs following both ICCE and ECCE. Likewise Gee and Tabbara ${ }^{10}$ have recently reported on a series of 15 patients with ECCE with no major intraoperative or postoperative complications. Ten of these patients had capsular fixated posterior chamber IOL lens implantation. In all 15 patients postoperative visual acuity was $6 / 12$ or better.

Jones $^{11}$ recently reported his findings from a series of patients with FHIC having ECCE with or without posterior chamber IOLs. He found that anterior chamber haemorrhage was common during surgery, that significant uveitis was present in $25 \%$ of patients with IOLs but absent in patients without IOLs, and that a rise in IOP occurred in $33 \%$ of eyes. Vitreous opacities were frequently present but visual acuity was $6 / 12$ or better in $70 \%$ of eyes with IOLs and $80 \%$ of eyes corrected with contact lenses. We have found haemorrhage following paracentesis to be invariable in FHIC. This haemorrhage appears to be caused by lowering the IOP, and the effects may be minimised by the use of sodium hyaluronate before performing anterior capsulotomy. We observed no postoperative hyphaema. This may have been due to effective wound closure avoiding postoperative hypotony. Postoperative uveitis occurred in four eyes $(22 \%)$ but unlike Jones ${ }^{11}$ we did not find any association with IOL use.

Preoperative treatment with systemic steroids may reduce postoperative uveitis as has been suggested in other uveitis syndromes. ${ }^{14}$ This was the case in three patients in this study. Although four eyes had raised IOP postoperatively only one eye has shown a sustained rise requiring more treatment than was needed before the surgery. The visual outcome in our series is similar to that of Jones' series " but slightly worse than in Gee's series. ${ }^{10}$ Though the number of patients achieving $6 / 12$ or better is high one would expect a higher number of $6 / 6$ or better eyes in these relatively young patients. This slight reduction in visual acuity appears to be caused by vitreous opacification which is known to be as common in FHIC as cataract itself. None of our patients has had vitrectomy for vitreous opacities but cataract surgery combined with vitrectomy for vitreous opacities may lead to a better visual result. Stellate KPs on both the IOLs and the posterior capsule were common but were not considered when making the decision whether to implant an IOL or not. 


\section{Conclusion}

Cataract extraction in patients with FHIC is only slightly more difficult than in patients with senile cataract. Efforts should be made to minimise postoperative uveitis by preoperative treatment with steroids systemically or locally. Visual outcome is often slightly compromised due to persistent vitreous opacities. IOL implantation in this series does not appear to be associated with increased risk and in view of the poor compliance with contact lens wear should be considered in the patient with unilateral FHIC.

This study was supported in part by the Anne Allerton Fund.

1 Fuchs E. Ueber Komplikationer de Heterochromie. Z Augenheilkd 1906; 15: 191-212.

2 Liesegang TJ. Clinical features and prognosis in Fuchs' uveitis syndrome. Arch Ophthalmol 1982: 100: 1622-6.

3 Kimura SJ, Hogan MJ, Thygeson P. Fuchs' syndrome of heterochromic cyclitis. Arch Ophthalmol 1955; 54: 179-86.
4 Mooney D, O'Connor M. Intraocular lenses in Fuchs' heterochromic cyclitis. Trans Ophthalmol Soc UK 1980; 100: 510.

5 Mills KB, Rosen ES. Intraocular lens implantation following cataract extraction in Fuchs' heterochromic uveitis. Ophthalmic Surg 1982; 13: 467-9.

6 Ward DM, Hart CT. Complicated cataract extraction in Fuchs' heterochromia. Brf Ophthalmol 1967; 51: 530-8.

7 Smith RE, O'Connor GR. Cataract extraction in Fuchs' syndrome. Arch Ophthalmol 1974; 91: 39-41.

8 Jain IS, Gupta A, Gangwar DN, Dhir SP. Fuchs' heterochromic cyclitis: some observations on clinical picture and chromic cyclitis: some observations on clinical picture

9 Norn MS. Cataract extraction in Fuchs' heterochromia. Acto Ophthalmol (Kbh) 1968; 46: 685-99.

10 Gee SS, Tabbara KF. Extracapsular cataract extraction in Fuchs' heterochromic iridocyclitis. Am $\mathcal{F}$ Ophthalmol 1989; 108: $310-4$.

11 Jones NP. Extracapsular cataract surgery with and without intraocular lens implantation in Fuchs' heterochromic uveitis. Eye 1990; 4: 145-50.

12 Tabbutt BR, Tessler HH, Williams D. Fuchs' heterochromic iridiocyclitis in blacks. Arch Ophthalmol 1988; 106: 1688-90.

13 O'Connor DR. Heterochromic iridocyclitis. Trans Ophthalmol Soc UK 1985; 104: 219-31.

14 Foster CS, Fong LP, Sing G. Cataract surgery and intraocular lens implantation in patients with uveitis. Ophthalmology lens implantation 